\title{
LES study on mixing and combustion in a Direct Injection Spark Ignition engine
}

\author{
Nicolas Iafrate ${ }^{1, *}$, Anthony Robert ${ }^{1}$, Jean-Baptiste Michel ${ }^{1}$, Olivier Colin ${ }^{1}$, Benedicte Cuenot $^{2}$, \\ and Christian Angelberger ${ }^{1}$ \\ ${ }^{1}$ IFP Energies nouvelles, 1-4, avenue de Bois-Préau, 92852 Rueil-Malmaison Cedex, France \\ ${ }^{2}$ CERFACS, 42 avenue Gaspard Coriolis, 31000 Toulouse, France
}

Received: 26 February 2018 / Accepted: 27 June 2018

\begin{abstract}
Downsized spark ignition engines coupled with a direct injection strategy are more and more attractive for car manufacturers in order to reduce pollutant emissions and increase efficiency. However, the combustion process may be affected by local heterogeneities caused by the interaction between the spray and turbulence. The aim for car manufacturers of such engine strategy is to create, for mid-to-high speeds and mid-up-high loads, a mixture which is as homogeneous as possible. However, although injection occurs during the intake phase, which favors homogeneous mixing, local heterogeneities of the equivalence ratio are still observed at the ignition time. The analysis of the mixture preparation is difficult to perform experimentally because of limited optical accesses. In this context, numerical simulation, and in particular Large Eddy Simulation (LES) are complementary tools for the understanding and analysis of unsteady phenomena. The paper presents the LES study of the impact of direct injection on the mixture preparation and combustion in a spark ignition engine. Numerical simulations are validated by comparing LES results with experimental data previously obtained at IFPEN. Two main analyses are performed. The first one focuses on the fuel mixing and the second one concerns the effect of the liquid phase on the combustion process. To highlight these phenomena, simulations with and without liquid injection are performed and compared.
\end{abstract}

\section{Introduction}

In the last decade, downsized Direct Injection Spark Ignition (DISI) engines have been developed by the majority of automotive manufacturers for their potential to significantly reduce the fuel consumption and pollutant emissions. However, they raise a number of technical challenges such as abnormal combustion phenomena (i.e., pre-ignition or knock [1]), and particulate emissions especially during engine transient operation and cold-start. The use of direct injection allows to cool the air inside the combustion chamber and reduce the risk of abnormal combustion. Nevertheless, direct injection, even taking place early in the cycle, can create equivalence ratio and temperature stratifications in the combustion chamber and therefore affect ignition and combustion for mid-to-high speeds and mid-up-high loads [2]. A detailed analysis of these stratifications close to the Spark Timing (ST) is experimentally complex [3]. While RANS approach is limited to the description of the mean cycle and consequently is not able to reproduce cycle-to-cycle variation, Large-Eddy Simulation (LES) can provide a detailed insight of the coupling

\footnotetext{
* Corresponding author: nicolas.iafrate@ifpen.fr
}

between injection, turbulence and combustion, thanks to its unique capability to reproduce highly transient and turbulent phenomena [4].

For several years, many numerical studies have been investigated the occurence and causes for variability of spark ignition engines by LES, such as in-cylinder mixture composition or internal aerodynamics. Fontanesi et al. [5, 6] used several LES cycles to better understand the knock phenomenon. Liquid injection modeling increases understanding of cyclic combustion variability. For example, a study of aerodynamics/spray interactions is proposed in the work of Adomeit et al. [7] showing the need to take into account the liquid injection. The authors showed that there is a significant increase in kinetic energy of the cyclic fluctuations with fuel injection and it also provided different equivalence ratio fields around the spark plug. Another study of Forte et al. [8] showed that the combustion is much more sensitive to small perturbations of the equivalence ratio near the park plug which impact the laminar flame speed.

The present study adresses a LES study of the influence of liquid injection in a DISI engine. Beyond the comparison with the exprimental data, analysis of the stratifications due to the liquid injection is performed in the whole combustion chamber and around the spark plug. Comparisons 
with previous calculations, assuming a fully homogeneous mixing, are also done in order to highlight which quantities are impacted by the stratifications during the combustion.

\section{Numerical models}

Large Eddy Simulations (LES) are conducted with the AVBP solver [9, 10], co-developed and co-owned by CERFACS and IFPEN. AVBP solves the multi-species, compressible, reactive Navier-Stokes equations on unstructured and moving meshes. It includes a Lagrangian solver for the description of spray dynamics [11]. In this section, the models used for liquid injection, ignition and turbulent combustion are recalled.

\subsection{Injection modeling}

In the Lagrangian formalism applied here, the point-source approximation is used which implies a dilute spray and droplets smaller than flow characteristic scales. In engine flow conditions, the only force acting on droplets is drag [12]. In the present work, the sub-grid scale contribution to drag is not taken into account.

Liquid injection is described with the GDI-model (Gaussian Disk Injection model [13]) which assumes that liquid at the nozzle exit is already in the form of a spray, omitting the dense region of the liquid jet [14,15]. With this assumption, droplets are injected at random positions over a disk surface $S_{\mathrm{inj}}$ located at the nozzle exit. The droplet size distribution at injection follows a Rosin-Rammler [16] distribution, with a Sauter mean diameter equal to $8 \mu \mathrm{m}$ and a standard deviation of 2.2. The mean droplet injection velocity $V(r)$ follows a Gaussian radial profile [17] of the form:

$$
V(r)=V_{\max } \exp \left(-\sigma \frac{r^{2}}{R^{2}}\right)
$$

where $R$ is the radius of the injection disk and $\sigma$ the thickness of the Gaussian profile. As the droplet size distribution does not depend on $r$, the maximum velocity $V_{\max }$ is related to the mean injection velocity $V_{\mathrm{inj}}$ as:

$$
V_{\max }=\frac{\sigma V_{\mathrm{inj}}}{1-\exp (-\sigma)}
$$

where $V_{\text {inj }}$ is defined from the injection volumic liquid flow rate $Q_{\mathrm{inj}}$ as:

$$
V_{\text {inj }}=\frac{Q_{\text {inj }}}{\alpha_{1} S_{\text {inj }}}
$$

where $\alpha_{1}$ is the cavitation coefficient, evaluated from a prior in-nozzle calculation as 0.68 . Note that the liquid flow rate evolves with time as illustrated in Figure 1a.

The parameter $\sigma$ is determined so as to limit the maximum velocity $V_{\max }$ to the Bernoulli velocity where $V_{\mathrm{B}}=\sqrt{2 \rho_{1} \Delta P}$ is the liquid fuel density and $\rho_{1}$ is the pressure drop in the injector.

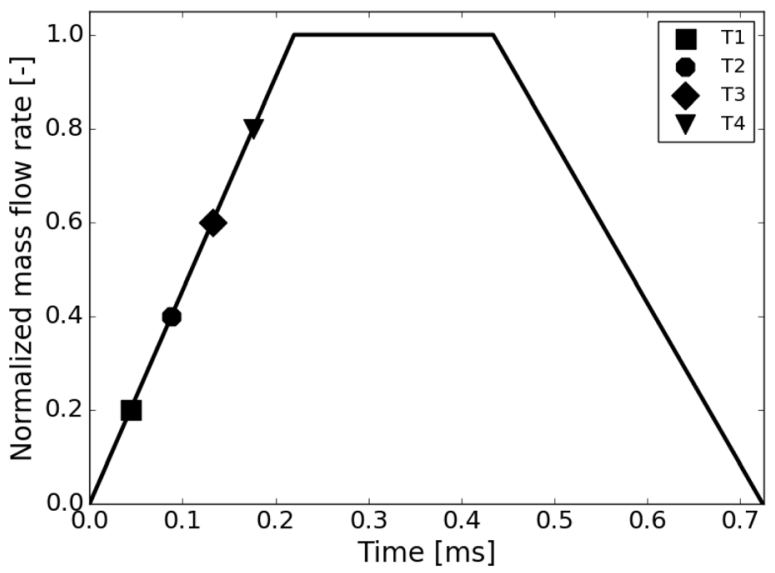

(a) Normalized injection rate

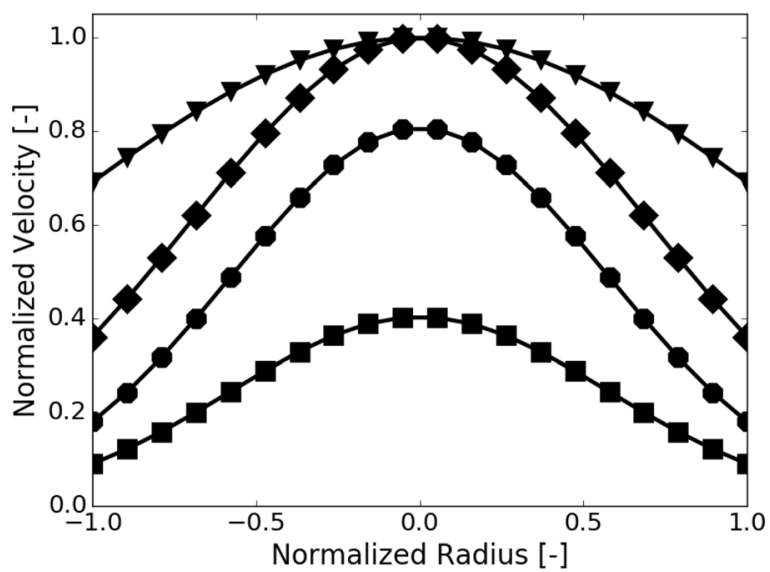

(b) Normalized velocity profile

Fig. 1. a. Normalized injection rate with time; b. injection velocity profiles at 4 instants marked with corresponding symbols on the plot in (a).

Finally, turbulent velocity fluctuations are introduced at injection by adding a random velocity contribution to the liquid velocity $V(r)$ :

$$
V(r)=V(r)\left(1+2 \mathscr{R} V_{\text {rms }}\right)
$$

with $\mathscr{R}$ and $V_{\text {rms }}$ a random number and a dimensionless turbulence intensity, set here at $20 \%$.

This model has been validated for two kind of injectors by [13] with experimental comparisons. Figure 1 b shows the temporal evolution of the injection velocity profile along the injector disk diameter.

\subsection{Turbulent combustion and ignition modeling}

The turbulent sub-grid scale stress tensor is modeled with the Sigma model [18] while the ESO2 hybrid numerical strategy [19] is used for space and time discretization. The fuel is represented by iso-octane. The choice of a single-component fuel in this study (for experiments and calculations) has been done to have well-known thermodynamic and chemical properties for calculations. To account for turbulent combustion in Spark Ignition (SI) engines, the flame 
Table 1. Main engine specifications.

\begin{tabular}{lcc}
\hline Parameter & Unity & Value \\
\hline Bore & {$[\mathrm{mm}]$} & 77 \\
Stroke & {$[\mathrm{mm}]$} & 85.8 \\
Speed & {$[\mathrm{RPM}]$} & 1800 \\
IMEP & {$[\mathrm{bar}]$} & 19 \\
Fuel & {$[-]$} & Isooctane \\
\hline
\end{tabular}

front propagation is described using the ECFM-LES premixed combustion model [20,21], without auto-ignition model. To represent physical phenomena describing ignition in DISI engine, the ISSIM-LES model [22] is used. The numerical model is divided in two parts: after an initial burned gases deposit, the flame kernel growth is modeled thanks to a modified flame surface density equation.

\section{Experimental configuration and numerical set-up}

The experimental configuration consists of a four-valve downsized single cylinder of $400 \mathrm{~cm}^{3}$ described in Table 1 .

The injector is a 6-holes asymmetric injector that minimizes fuel impingement on the valves and the combustion chamber walls (Fig. 2). Due to the spray targetting, no liquid film model is used in the simulations (which was not widely validated in engine conditions).

The injection operating conditions are reported in Table 2.

Figure 3 represents the computational domain of the LES. It includes the combustion chamber and the intake pipe in order to correctly capture the internal aerodynamics [23].

Due to the valves motion, 40 tetrahedral meshes are needed to simulate a full cycle. They contain from 3.0 to 12.4 million cells depending on the Crank Angle Degree (CAD). The mesh is refined around the valves to correctly describe the flow admitted or discharged. Cell size in the chamber is around $0.5 \mathrm{~mm}$ during the combustion phase and around $0.2 \mathrm{~mm}$ in the vicinity of the spark plug during ignition.

\section{Methodology}

As computing large numbers of consecutive cycles with LES is extremely costly, the present study benefited from a previous work by Robert et al. [1, 24, 25] where 30 consecutive cycles of a single cylinder DISI engine were computed. These computations however did not include direct liquid fuel injection, replaced by a perfectly premixed gaseous injection at the mean equivalence ratio of 1 .

In order to study the impact of liquid fuel injection at a reduced computational cost, only 5 different non-consecutive engine cycles (C1-C5) of the 30 were computed with the spray. The methodology is summarized in Figure 4: for each selected cycle, the solution of the gaseous case
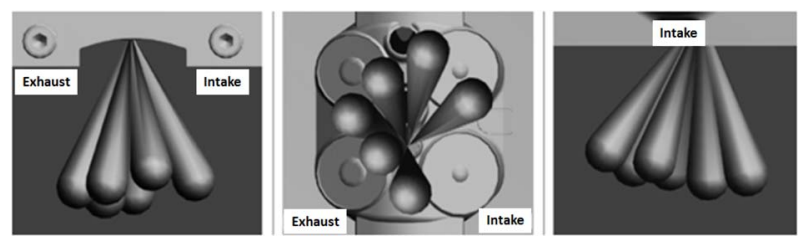

Fig. 2. 3D virtual representation of the 6-holes asymmetric injector.

Table 2. Injection operating conditions.

\begin{tabular}{lcc}
\hline Parameter & Unity & Value \\
\hline Start Of Injection(SOI) & {$[\mathrm{CAD}]$} & 77 \\
Injected mass & {$[\mathrm{mg}]$} & $51.2 \mathrm{~s}$ \\
Injection duration & {$[\mathrm{ms}]$} & 3.8 \\
Injection pressure & {$[\mathrm{bar}]$} & 200 \\
\hline
\end{tabular}

previously calculated is taken during the air admission phase (i.e., just before fuel injection) and used as the initial condition for the present calculation including liquid fuel injection. Then the LES is run with the liquid fuel injection to simulate the intake, compression and combustion phases. The aim of the method is to ensure the same pressure and air trapped mass, thereby the fresh gases temperature is decreased.

The 5 non-consecutive simulated cycles have be chosen so as to be statistically independent. With this methodology, it is now possible to directly compare cycles with and without liquid injection. Note that the present methodology is valid only if liquid fuel injection does not introduce any dependency between consecutive cycles. This is justified in the present case by the early injection timing.

\section{Results and discussions}

Figure 5 shows the evolution of the liquid mass in the chamber during the intake and compression phases. The liquid fuel is totally evaporated before the Top Dead Center (TDC, corresponding to a crank angle), and in particular at sparking time which occurs at 6.5 CAD After the Top Dead Center (ATDC). Therefore the spray does not directly interact with ignition or combustion, and its effect is only due to induced temperature and fuel vapor heterogeneities.

In this section, the impact of liquid injection on the temperature and equivalence ratio distribution of the unburnt gas is first analyzed. Then the influence of this modified fresh gas state on the turbulent flame propagation is studied.

\subsection{Impact of liquid injection on the fresh gases state}

Droplet evaporation introduces local heat and mass transfers that have however different impacts on the mixture. These are studied in the next sections. 


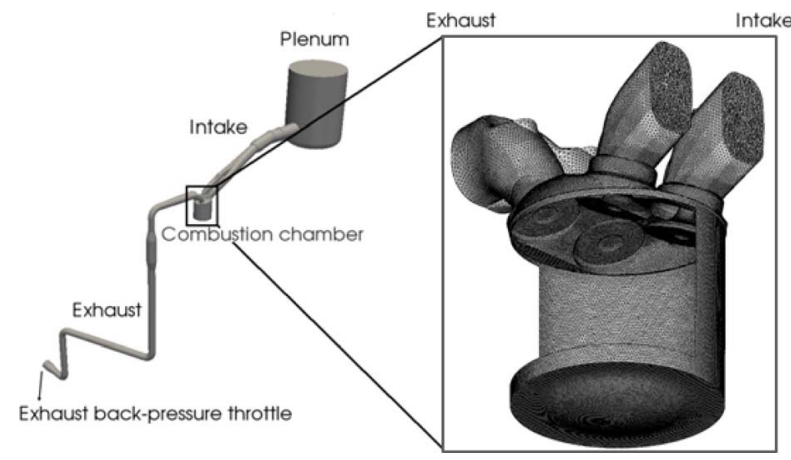

Fig. 3. LES computation domain and a mesh of the combustion chamber.

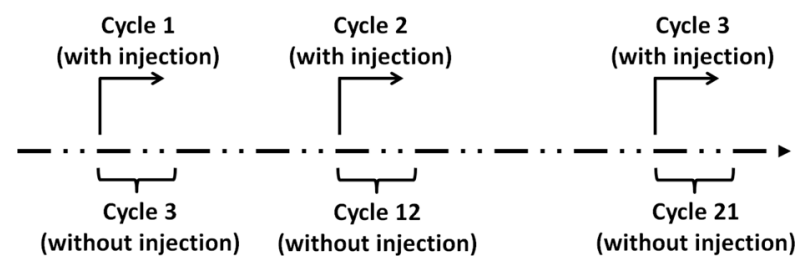

Fig. 4. LES methodology to compute intake, compression and combustion with liquid fuel injection: each cycle is restarted from the previous gaseous calculation of Robert et al. [1, 24, 25].

\subsubsection{Effect on the fresh gases temperature}

One major effect of liquid injection in DISI engines is air cooling inside the combustion chamber as a result of evaporation of the liquid fuel. This reduces the risk of abnormal combustion, and could allow higher compression ratios. The global effect of fuel evaporation on the mixture temperature can be estimated by an average temperature variation $\Delta T$, neglecting heat losses, as:

$$
\Delta T \approx \frac{m_{1} L_{\mathrm{v}}}{m_{\mathrm{g}} C_{\mathrm{v}}}
$$

where $m_{\mathrm{l}}$ and $m_{\mathrm{g}}$ are the liquid and gas mass in the combustion chamber, $L_{\mathrm{v}}$ is the latent heat of fuel vaporization, and $C_{\mathrm{v}}$ is the specific heat at constant volume of the gaseous mixture. For the simulated conditions, the above expression yields a temperature decrease of $\Delta T=25.7 \mathrm{~K}$, which is moderate in comparison with the temperatures reached during ignition and combustion.

Figure 6 shows the time evolution of the volume averaged in-cylinder temperature until sparking for the 5 cycles. Both simulations, with and without liquid injection are shown. No cyclic variability of this integral quantity is observed, leading to exactly superposed curves for all cycles. Liquid injection leads to a decrease of $25 \mathrm{~K}$ of the mean gas temperature, i.e., very close to the estimation of equation (5).

To further highlight the impact of liquid fuel evaporation on the mixture temperature, Figures 7 and 8 show the Probability Density Function (PDF) of the fresh gases temperature in the combustion chamber respectively at the end of liquid evaporation (-50 CAD) and before sparking

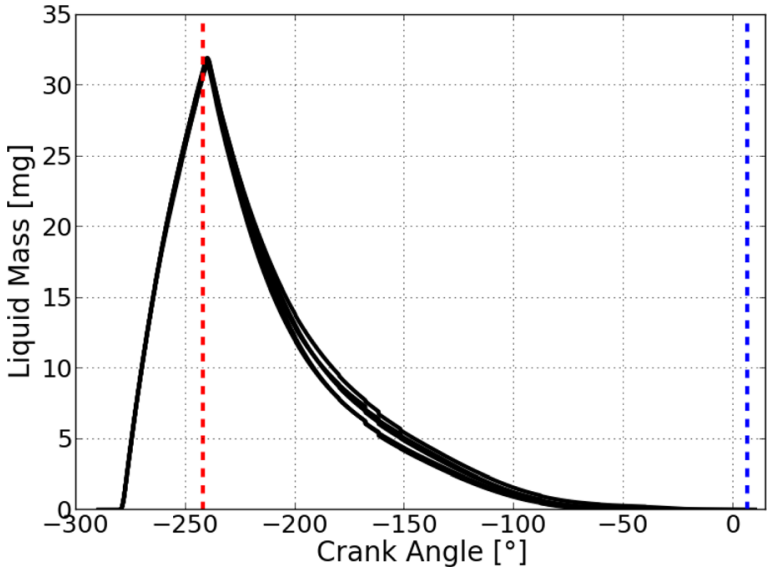

Fig. 5. Liquid fuel evolution during the intake and compression phases for the 5 cycles. The red and blue dashed lines represent respectively the end of injection and the spark timing.

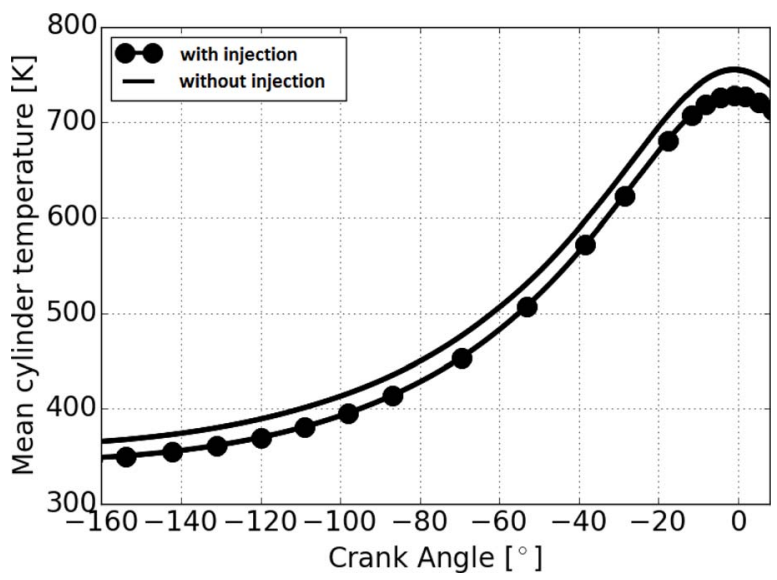

Fig. 6. Mean cylinder temperature evolution for the 5 cycles with and without injection. All 5 curves merge in one as no variability is observed for this quantity.

(5 CAD) for cycles C2. Similar distributions were obtained for all cycles, therefore results for only one cycle are presented.

It appears clearly that liquid injection leads to a much wider statistical distribution of the fresh gases temperature, i.e., a higher stratification. In the gaseous case, wall heat losses are the only source of temperature stratification, which stays very limited. At $-50 \mathrm{CAD}$, corresponding to the end of liquid fuel evaporation in the liquid injection case, the width of the PDF is around $40 \mathrm{~K}$ in the gaseous case while it reaches $100 \mathrm{~K}$ with liquid injection. Before sparking, the width of the PDF has increased in both cases because of mixing, but the PDF of the gaseous case still exhibits a very marked peak at $750 \mathrm{~K}$, contrary to the liquid injection case where no peak is visible.

The temperature stratification resulting from fuel evaporation is directly linked to the equivalence ratio heterogeneities, as rich regions correspond to higher evaporation and therefore colder temperature. This is confirmed in Figures 9 and 10 also showing the PDF of the mixture 


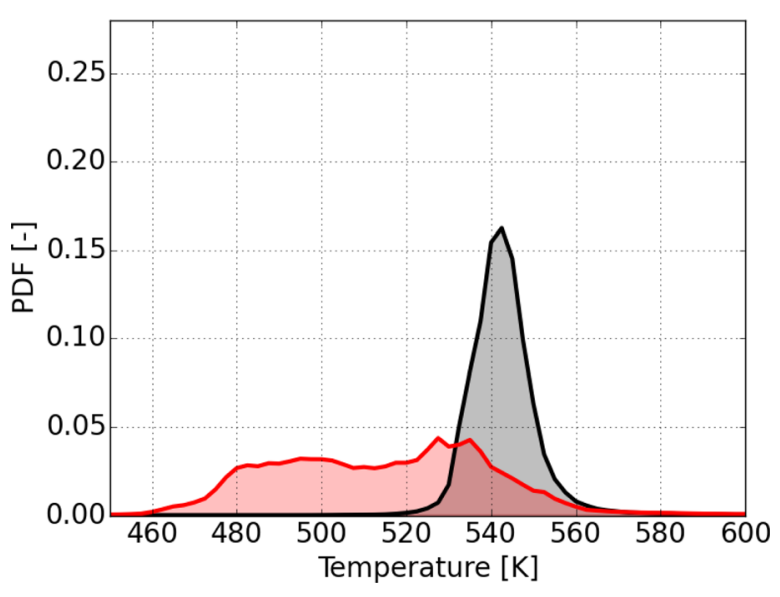

Fig. 7. PDF of gases temperature at the end of liquid evaporation ( $-50 \mathrm{CAD})$ in the combustion chamber for cycle C2. Black: without liquid injection; Red: with liquid injection.

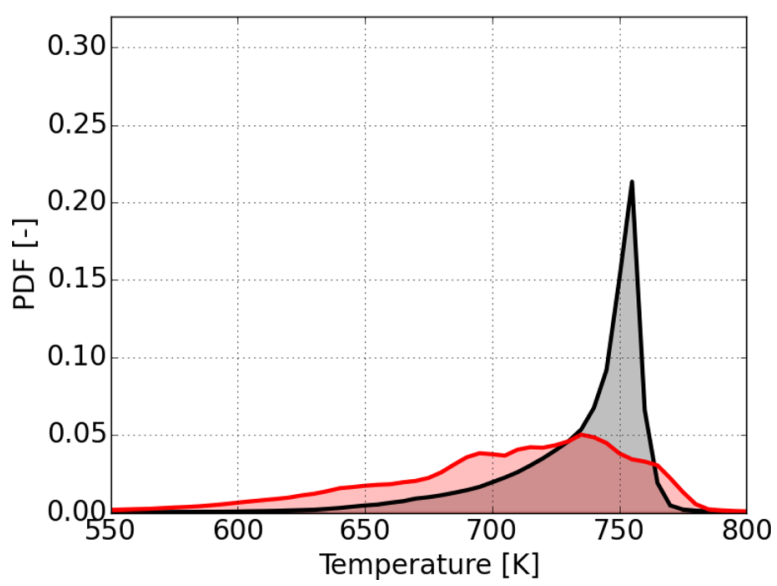

Fig. 8. PDF of gases temperature before sparking (5 CAD) in the combustion chamber for cycle C2. Black: without liquid injection; Red: with liquid injection.

temperature conditioned on the equivalence ratio $\varphi$ being in the range $0.95<\varphi<1.05$ for the case with liquid injection. The conditioned PDF is much narrower than the unconditioned one, and comparable to the PDF observed without liquid injection. Note also the difference of $25 \mathrm{~K}$ between the peak temperatures of the conditioned PDF with liquid injection and the PDF without liquid injection, i.e., the same difference between the average temperatures of Figure 6. This confirms that evaporation is the main responsible for the increased temperature stratification of the liquid injection case. The temperature stratification, due to evaporation, remains moderate compared to the igniting and burning temperatures and its effect on the cycle evolution will be merely visible. However it may locally modify the flame speed as will be shown later.

Evaporation is also associated to a stratification of the equivalence ratio, which is critical for ignition and combustion and is studied in the next section.

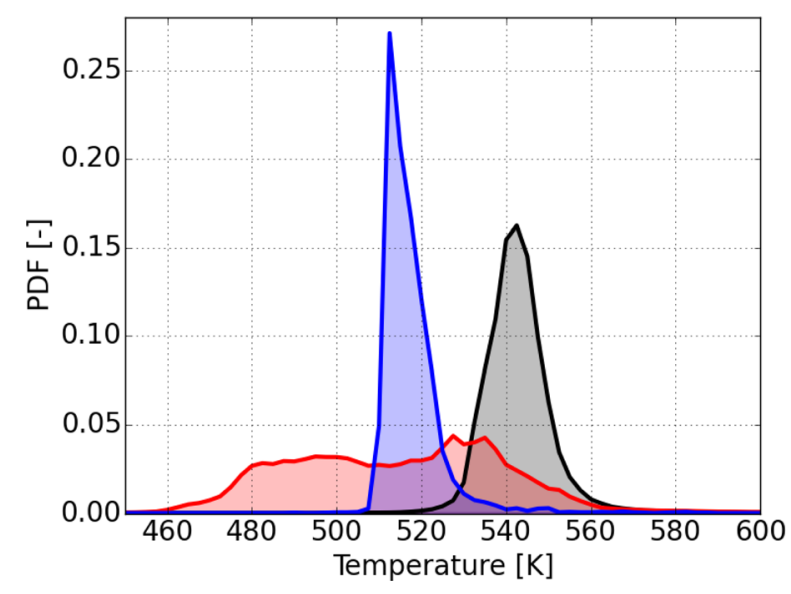

Fig. 9. PDF of gases temperature at the end of liquid evaporation $(-50 \mathrm{CAD})$ in the combustion chamber for cycle C2. Black: without liquid injection; Red: with liquid injection; Blue: with liquid injection, conditioned on $0.95<\varphi<1.05$.

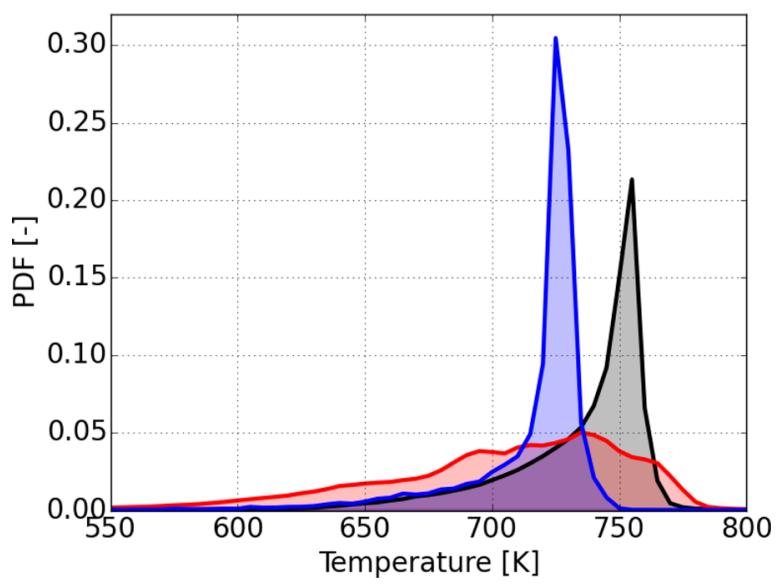

Fig. 10. PDF of gases temperature before sparking (5 CAD) in the combustion chamber for cycle C2. Black: without injection; Red: with liquid injection; Blue: with liquid injection, conditioned on $0.95<\varphi<1.05$

\subsubsection{Effect on the equivalence ratio}

Figures 11 and 12 show the PDFs of the equivalence ratio in the combustion chamber for cycles $\mathrm{C} 2$ and $\mathrm{C} 4$ with liquid injection at three instants: End Of Injection (EOI), End Of liquid Evaporation (EOE) and at Sparking Time (ST). For both cycles, heterogeneities are very important at the end of injection because the injected spray is not yet fully evaporated and the evaporated fuel is not yet well mixed with air. This leads to high local values of equivalence ratio inside the spray which is clearly visible on Figure 13 showing the fuel vapor concentration during the injection phase: mixing with the surrounding air is limited, leading to the observed high local equivalence ratios.

At the end of evaporation (i.e. $190 \mathrm{CAD}$ after EOI) the tumble motion enhanced mixing, decreasing heterogeneities in the combustion chamber which reflects on the PDFs of Figures 11 and 12 by a width reduction down to around 


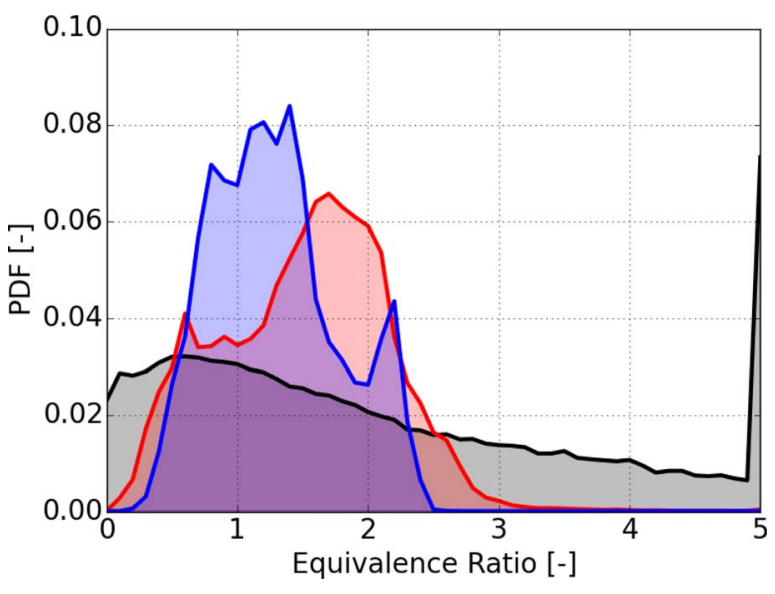

Fig. 11. PDF of the equivalence ratio in the combustion chamber for cycle $\mathrm{C} 2$ with liquid injection at 3 instants: end of injection (Black); end of evaporation (Red); sparking time (Blue).

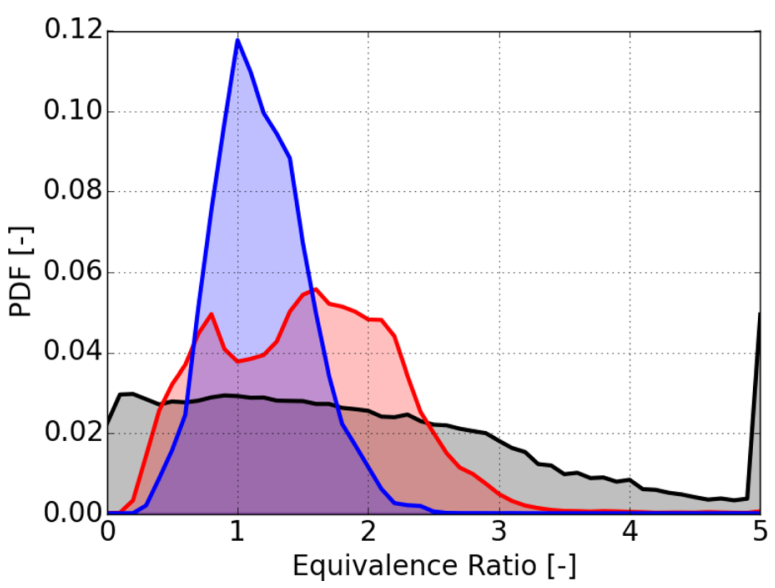

Fig. 12. $\mathrm{PDF}$ of the equivalence ratio in the combustion chamber for cycle $\mathrm{C} 4$ with liquid injection at 3 instants: end of injection (Black); end of evaporation (Red); sparking time (Blue).

3 in equivalence ratio. At that time, note that fuel vapor is present in the whole chamber. At ST, the mixing process has further homogenized the mixture and PDFs are now centered at 1 . However heterogeneities are still present, as demonstrated by the PDF widths around 2.5 , i.e., not much reduced in comparison to the PDFs at the end of evaporation. Cycle C4 (Fig. 12) seems better mixed, as the PDF exhibits only one peak at this time, while for cycle C2 (Fig. 11) the multiple peaks of the PDF, in particular on rich values, indicate more stratification.

To focus more about stratification at ignition, a similar analysis is performed in the volume defined by a $2 \mathrm{~cm}$ diameter sphere around the spark plug position. Results are shown in Figures 14 and 15 for cycles $\mathrm{C} 2$ and $\mathrm{C} 4$ respectively. Overall the same comments made above for the whole combustion chamber still hold and the mixture is clearly heterogeneous even in such a small volume.

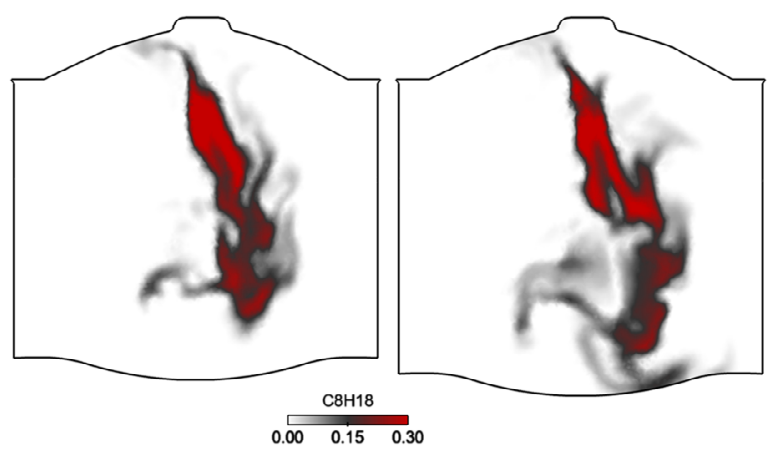

Fig. 13. Instantaneous field of fuel vapor mass fraction in a central vertical plane of the chamber for cycle $\mathrm{C} 2$, at 21 and 26 CAD after SOI.

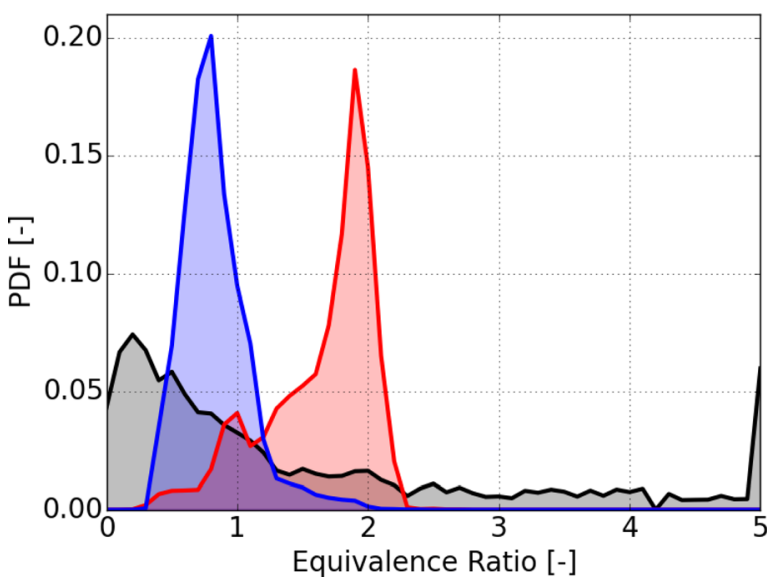

Fig. 14. PDF of equivalence ratio around the spark plug for cycle $\mathrm{C} 2$ at 3 instants: end of injection (Black); end of evaporation (Red); sparking time (Blue).

Important differences are also observed between the two cycles: at the sparking time, the PDF of cycle $\mathrm{C} 4$ is centered on 1 , whereas the PDF of cycle $\mathrm{C} 2$ is centered on 0.8 . These differences already appeared earlier at the end of liquid evaporation, where the PDF for cycle $\mathrm{C} 4$ is quite broad with a most probable value of equivalence ratio around 2.2 , while for cycle $\mathrm{C} 2$ less stratification and a leaner peak value around 1.8 are observed.

As will be shown later, such mixture heterogeneities around the spark position lead to different flame ignition and propagation behaviors compared to cases without liquid injection, and to a quite high variability between cycles as the $\mathrm{C} 2$ and $\mathrm{C} 4$ discussed here. The corresponding spatial distributions of $\varphi$ are illustrated on Figure 16 where instantaneous snapshots of the equivalence ratio field is shown in a horizontal cut plane at three different instants. The high grid resolution of the LES allows an accurate description of mixing, which results from the interactions of vortices of different sizes, generated by both the spray and the intake tumble motion. The visualizations of Figure 16 also confirm the strong heterogeneities still present close to ST for both cycles, even if cycle C2 appears slightly leaner and more homogeneous than cycle $\mathrm{C} 4$ in this plane of the combustion chamber. 


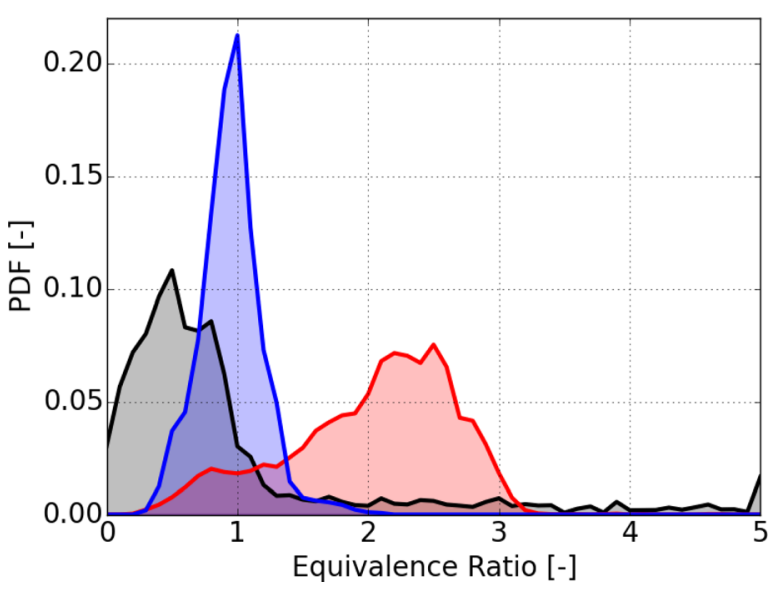

Fig. 15. PDF of equivalence ratio around the spark plug for cycle C4 at 3 instants: end of injection (Black); end of evaporation (Red); sparking time (Blue).

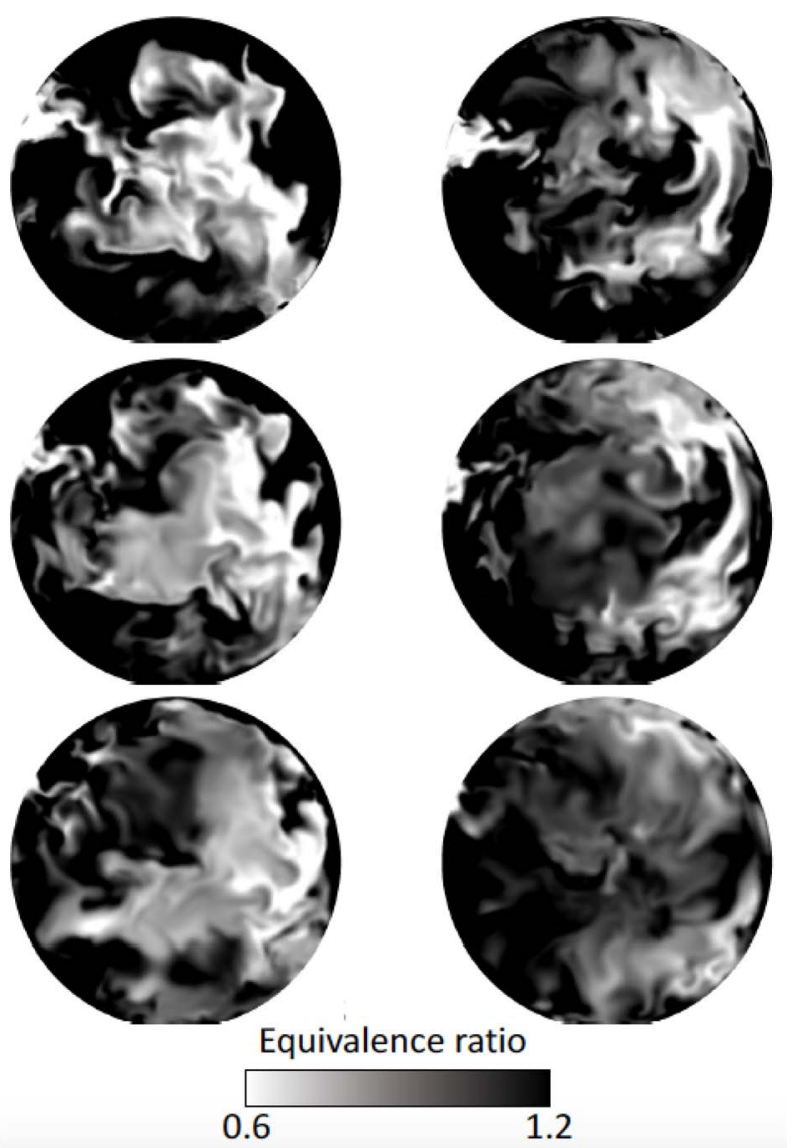

Fig. 16. Fields of equivalence ratio in a central horizontal cut plane through the cylinder below the spark plug at 10 (top), 20 (middle) and 30 (bottom) CAD after TDC for cycle C2 (left) and C4 (right).

Finally, Figure 17 displays the PDF of equivalence ratio conditioned on fresh gases in the whole combustion chamber for cycle $\mathrm{C} 2$ with liquid injection, at two crank angles during the burning phase. Similar qualitative observations have

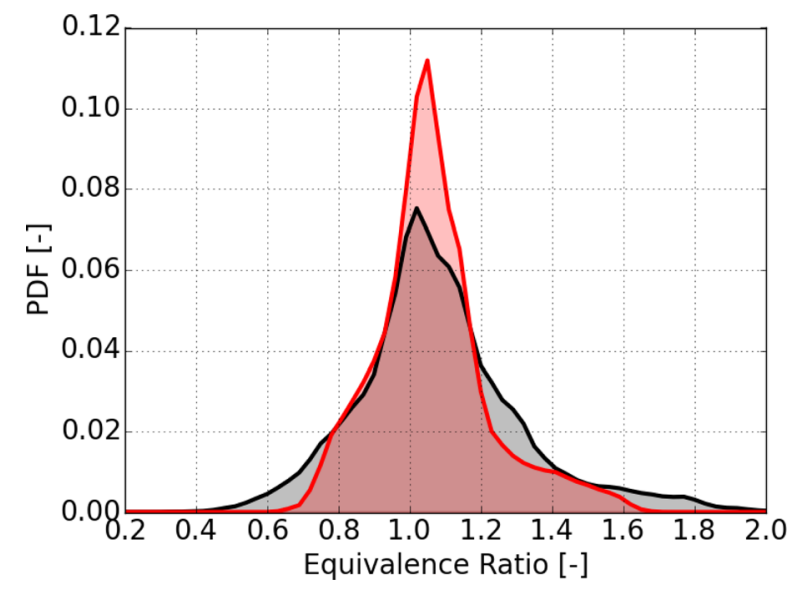

Fig. 17. PDF of equivalence ratio, conditioned on fresh gases, in the combustion chamber for cycle $\mathrm{C} 2$ at $20 \mathrm{CAD}$ (black) and 80 CAD (red) after TDC.

been obtained for all simulated cycles. At these times, the PDF peaks at the global equivalence ratio of 1 indicating that the whole chamber has been filled with fuel vapor in the same conditions, i.e., leading to uniform statistics. The equivalence ratio heterogeneity has reduced compared to the state at ignition, but is still there: the width of the $\mathrm{PDF}$ is now of the order of 1.4 and 1 , respectively for the two instants 20 and 80 CAD after TDC. This indicates a less efficient mixing after TDC, where the turbulence created during compression via the tumble motion gradually decreases in the absence of a turbulence source. Indeed the flame itself does not promote turbulence and mixing.

\subsection{Impact of direct liquid injection on combustion}

In this section the impact of the previously observed temperature and fuel stratification on the flame initiation and propagation is studied by comparison with results of LES without liquid injection. To ensure a reliable comparison, the exact same numerical parameters have been used in cases with and without liquid injection.

Figure 18 shows the mean cylinder pressure predicted by LES with and without liquid injection for the 5 selected cycles. Both series appear to be in the experimental envelope, even if cycle-to-cycle variation is slightly reduced with liquid injection. However, only 5 cycles are not sufficient to yield statistically reliable results and the cycle-to-cycle variation should be confirmed with more simulations. Despite the high heterogeneity of fresh gases temperature and equivalence ratio induced by liquid injection, it is noticeable that the global heat release rates and thus cylinder pressures are quite similar to cases without liquid injection.

Figures 19-21 illustrate the flame propagation for cycle C4 with and without liquid injection at respectively 10, 15 and 20 CAD after TDC. While the overall turbulent flame propagation is qualitatively similar for both cases, liquid injection leads to a more wrinkled flame surface, materialized by an iso-surface of the heat release rate. This suggests that the impact of stratification on the flame propagation may be compensated by the modified flame stretch induced 


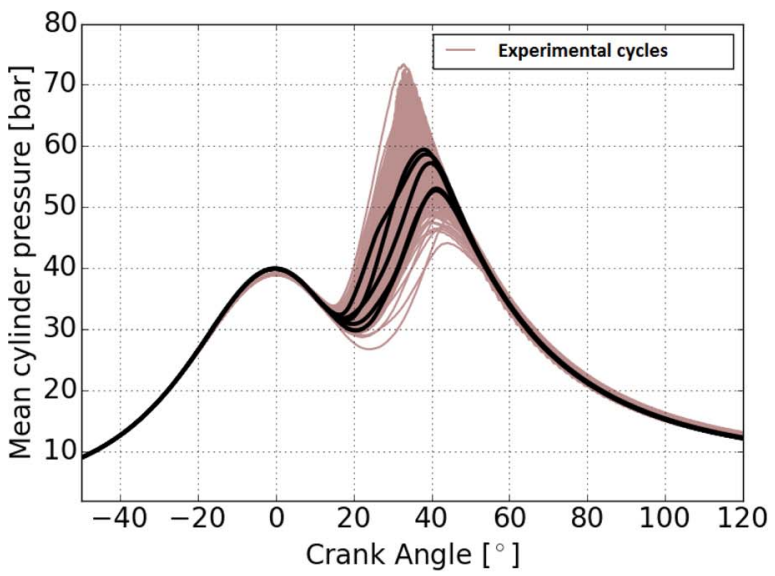

(a) with liquid injection

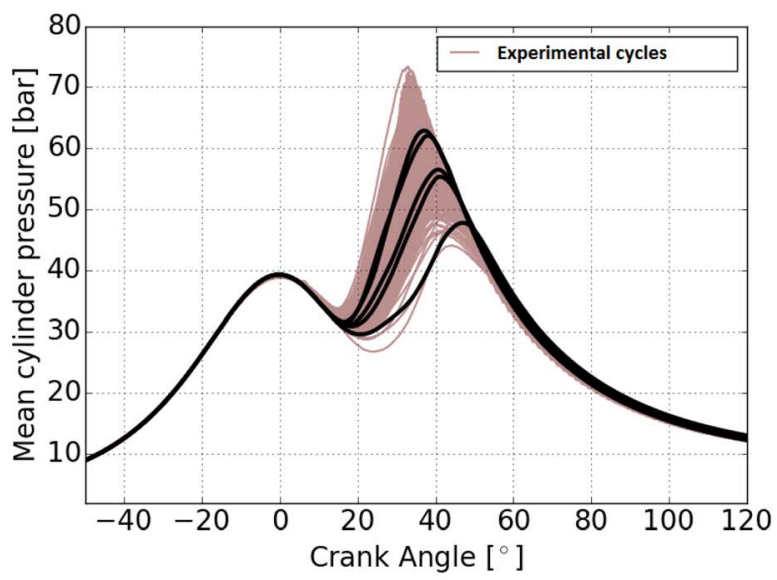

(b) without liquid injection

Fig. 18. Mean in-cylinder pressure comparison between LES (a) with and (b) without liquid injection (black) and experiment (brown).

by turbulence, leading to the similar global heat release rates of Figure 18. This mechanism is discussed below.

In the present LES, the ECFM-LES model [21, 22] is used to describe turbulent combustion. It is based on a Flame Surface Density approach, where the filtered reaction rate $\tilde{\tilde{\omega}}_{i}$ for species $i$ is modeled as follows:

$$
\bar{\rho} \tilde{\hat{\omega}}_{i}=\left.\rho^{u} \tilde{Y}_{i}^{u}\right|_{\mathrm{u}} S_{\mathrm{l}} \bar{\Sigma}
$$

where $\rho^{u}$ is the fresh gases density, $\bar{\Sigma}$ the filtered flame surface density, computed with a dedicated transport equation [21], $\left.\tilde{Y}_{i}^{u}\right|_{u}$ the resolved mass fraction of the species $i$ in the fresh gases and $S_{1}$ the local laminar flame speed calculated using the Metghalchi and Keck correlation [26]:

$$
S_{1}=S_{1}^{0}\left(\frac{T_{\mathrm{u}}}{T_{0}}\right)^{\alpha}\left(\frac{P_{\mathrm{u}}}{P_{0}}\right)^{\beta}
$$

where $S_{1}^{0}$ is the unstretched laminar flame speed at reference thermodynamic conditions $\left(T_{0}=298 \mathrm{~K}, P_{0}=1 \mathrm{~atm}\right)$ [9]. The coefficients $\alpha$ and $\beta$ are defined as a function of the local equivalence ratio, taking values of respectively 2.18 and -0.16 at stoichiometry. Figure 22 shows the laminar flame
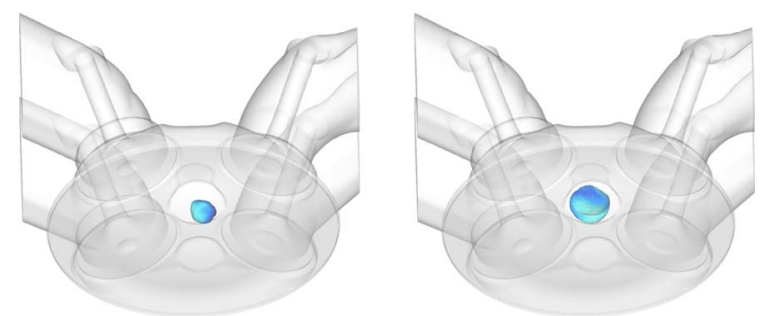

Fig. 19. Local heat release rate iso-surface for cycle $\mathrm{C} 4$ at 10 CAD after TDC - Left: without liquid injection; Right: with liquid injection.
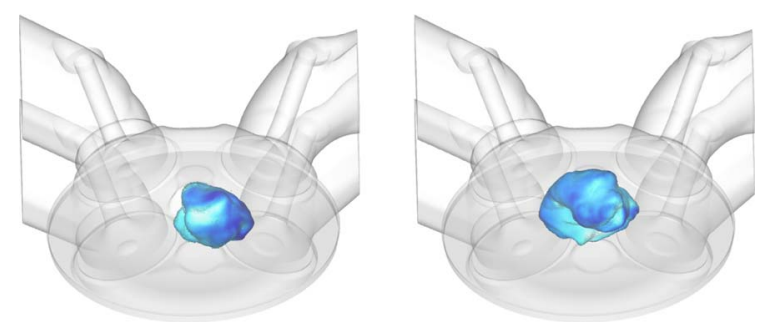

Fig. 20. Local heat release rate iso-surface for cycle $\mathrm{C} 4$ at 15 CAD after TDC - Left: without liquid injection; Right: with liquid injection.

speed evolution as a function of equivalence ratio for 3 different values of temperature, at a fixed pressure of 40 bar following the Metghalchi and Keck correlation [26].

The use of the Metghalchi and Keck correlation [26] allows to be consistent with the previous study of Robert et al. [1, 24, 25]. Others most precise methods can be found in the literature to estimate the laminar flame speed $[27,28]$.

The following sections address separately the impact of liquid injection on the local flame speed and the turbulent flame area.

\subsubsection{Impact on laminar flame speed}

The impact on the laminar flame speed of the fresh gas temperature can be estimated by introducing the temperature deviation $\Delta T_{\mathrm{u}}$, leading to the flame speed deviation $\Delta S_{\mathrm{l}}$ :

$$
\frac{\Delta S_{1}}{S_{1}}=\alpha \frac{\Delta T_{\mathrm{u}}}{T_{\mathrm{u}}}
$$

Taking as reference the fresh gas temperature at TDC with liquid injection $T_{\mathrm{u}}=725 \mathrm{~K}$ (Fig. 6), and a temperature deviation of $25 \mathrm{~K}$, equation (8) leads to a laminar flame speed deviation of $7.5 \%$. The laminar flame speed can be compared to the mean consumption flame speed of the turbulent flame, defined as the ratio of the total fuel consumption to the total flame surface area in the chamber. Consumption speeds obtained with and without liquid injection are compared in Figure 23 for cycles C2 and C4.

The presence of a fuel spray leads to a smaller mean consumption speed, decreased by around $25 \%$ compared to gaseous simulation. This is much higher than the $7.5 \%$ 

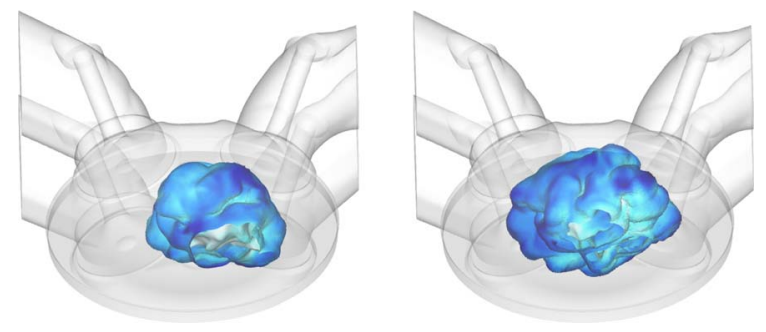

Fig. 21. Local heat release rate iso-surface for cycle $\mathrm{C} 4$ at 20 CAD after TDC - Left: without liquid injection; Right: with liquid injection.

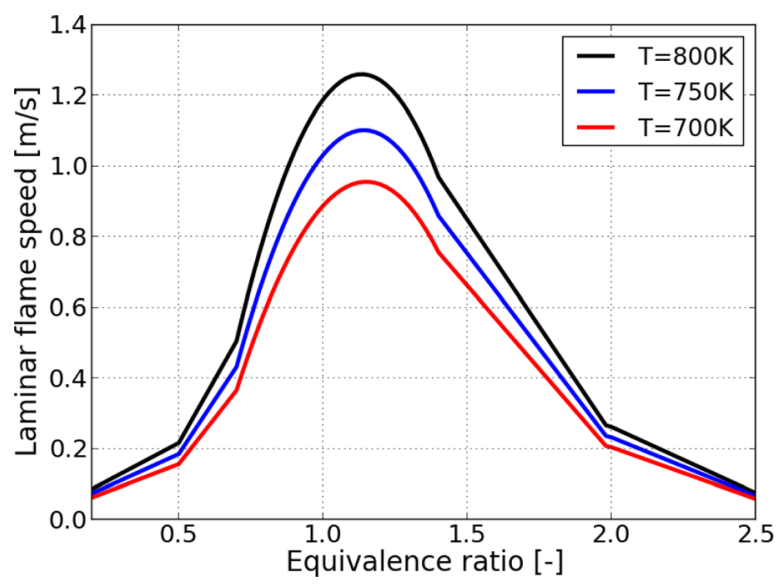

Fig. 22. Evolution of the laminar flame speed for three temperature $(P=40$ bar $)$.

estimation for the impact of fresh gas temperature fluctuation, and indicates a much stronger effect of equivalence ratio stratification. Moreover the slower consumption speed of cycle $\mathrm{C} 2$ is linked to the leaner mixture at the spark plug (Fig. 14), which is also consistent with the previous assessment.

Figure 24 shows the PDF of the local laminar flame speed computed with equation (7) in the combustion chamber at sparking time, taking the temperature, pressure and equivalence ratio from cycle $\mathrm{C} 2$ with and without liquid injection. To confirm the link between equivalence ratio stratification and flame speed, a third PDF is shown corresponding to the laminar flame speed computed with equation (7) taking now a constant equivalence ratio. The global impact of liquid injection on the local flame speed is clearly evidenced, leading to a wide range of values from $0.3 \mathrm{~m} / \mathrm{s}$ to $1.0 \mathrm{~m} / \mathrm{s}$, i.e., below the stoichiometric value, while the case with a fully homogeneous mixture exhibits a narrow peak at the stoichiometric value of $1.05 \mathrm{~m} / \mathrm{s}$. Ignoring the fluctuations of equivalence ratio and keeping only the temperature stratification leads to a narrowed PDF ranging from $0.5 \mathrm{~m} / \mathrm{s}$ to $1.1 \mathrm{~m} / \mathrm{s}$, i.e., slightly shifted toward stoichiometry.

This shows that the temperature and equivalence ratio stratifications caused by liquid injection have a comparable effect on the local consumption speed of the flame.

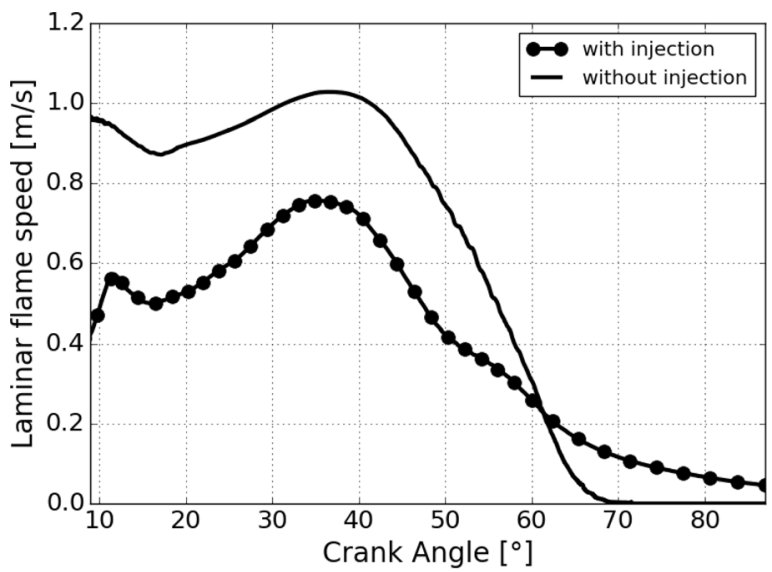

(a) Cycle $\mathrm{C} 2$

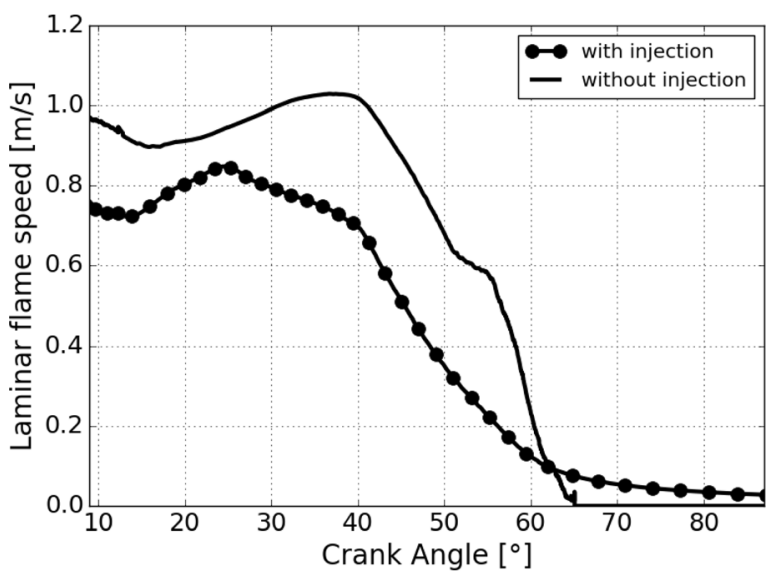

(b) Cycle $\mathrm{C} 4$

Fig. 23. Mean (averaged over the flame surface front) consumption speed for cycles $\mathrm{C} 2$ and $\mathrm{C} 4$ with and without liquid injection.

\subsubsection{Impact on flame surface}

A major factor impacting the local reaction rate of turbulent flame is the flame surface wrinkling. Figure 25 compares the total resolved flame surface with and without liquid injection for cycles $\mathrm{C} 2$ and $\mathrm{C} 4$. In contrast with previous findings on the laminar flame speed, liquid injection increases the flame area by up to $22 \%$. This is consistent with the more wrinkled flames observed in Figures 19-21. This increased wrinkling is a direct consequence of the variability of the flame consumption speed along the flame front, which propagates some parts of the flame faster than others. This leads to a deformation of the flame front, and eventually to increased wrinkling and flame area. Furthermore, the smaller mean laminar flame speed due to liquid injection increases the ratio $u^{\prime} / S_{1}$ [29], which implies that turbulence is more effective in wrinkling the flame front.

The two above mechanisms, i.e., local flame speed decrease and flame surface increase by liquid injection, therefore exhibit two opposite trends. Remembering that the reaction rate is proportional to the product of these two quantities (Eq. (6)), the impact of liquid injection on the total heat release rate and the subsequent cylinder 


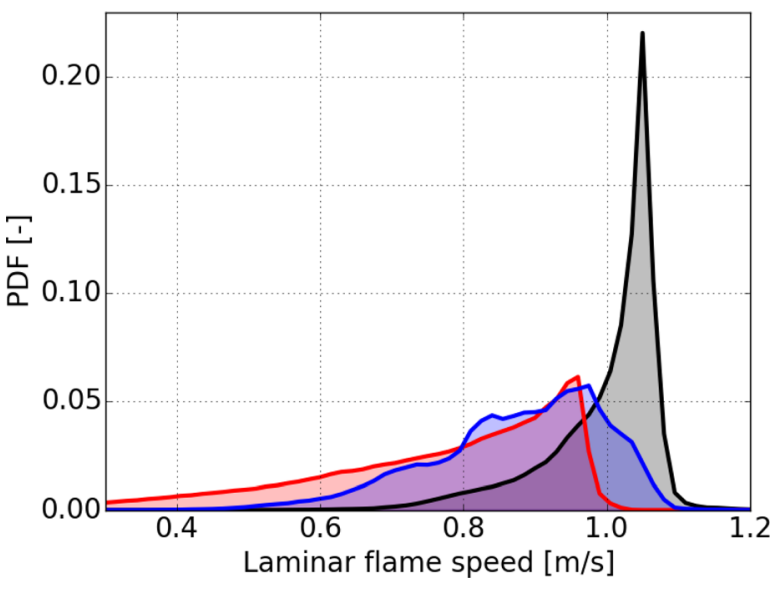

Fig. 24. PDF of the local laminar flame speed at sparking time for cycle C2 - Black: without liquid injection; Red: with liquid injection; Blue: with liquid injection, assuming uniform $\varphi=1$.

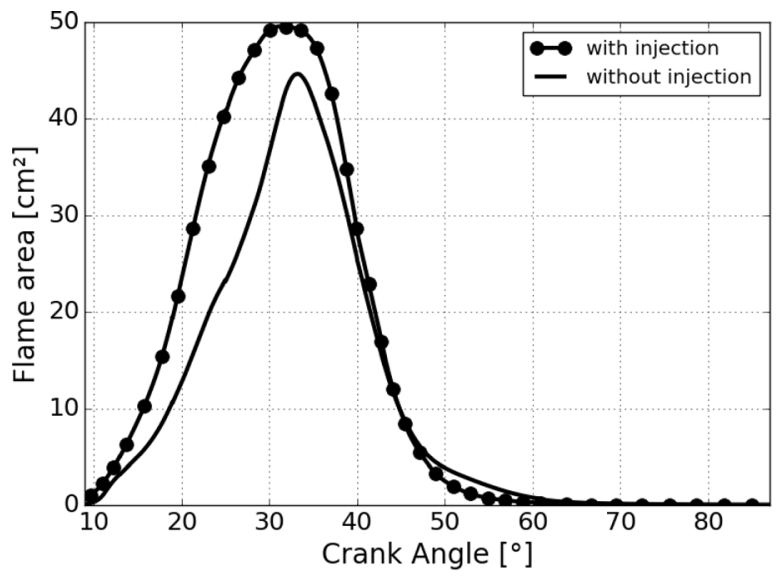

(a) Cycle 2

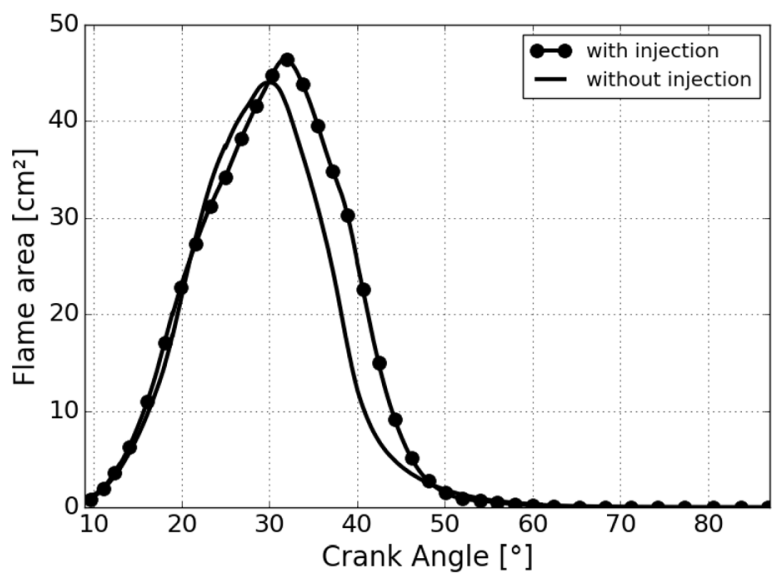

(b) Cycle 4

Fig. 25. Total resolved flame area for cycles $\mathrm{C} 2$ and $\mathrm{C} 4$ with and without liquid injection.

pressure is difficult to assess a priori. In the present case, it was shown in Figure 18 that the impact was in fact negligible, which means that both mechanisms tend to

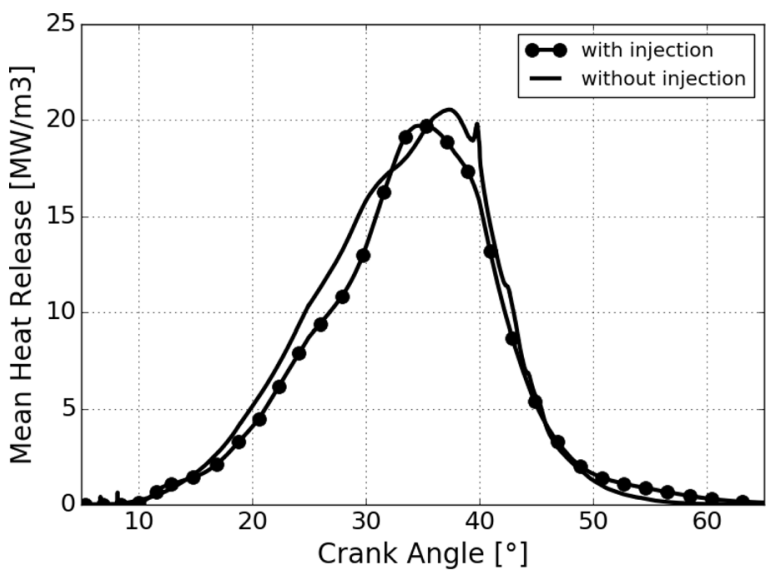

(a) Cycle 2

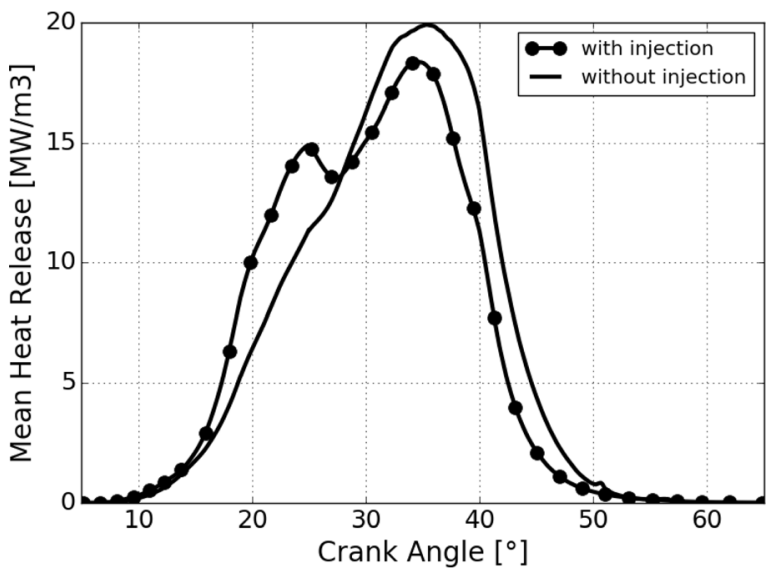

(b) Cycle 4

Fig. 26. Total heat release rate for cycles $\mathrm{C} 2$ and $\mathrm{C} 4$ with and without liquid injection.

compensate. To confirm this trend, Figure 26 shows the evolution of the total heat release rate during combustion for cycles $\mathrm{C} 2$ and $\mathrm{C} 4$ with and without liquid injection. The shape and levels of both curves are indeed very close for the two cycles.

The effect of the spray on mixing and the flame front appears to be significant and may moderate the sole effect of turbulence, which induces a high cycle-to-cycle variation linked to the tumble motion, explaining the lower variability observed in Figure 18.

\section{Conclusion}

This paper addresses an analysis of the impact of liquid injection on mixing and combustion in spark ignition engines. By comparing cycles with and without liquid injection computed with LES, it has been found that direct fuel injection not only cools down the fresh charge due to liquid evaporation, but also leads to very important inhomogeneities in temperature and equivalence ratio, that are kept until sparking and afterwards during the whole 
combustion phase. Despite this important fresh gases stratification, the overall combustion rate is surprisingly not much affected. This was explained by the counteracting effects of the spray on the local consumption speed, which is decreased, on one hand, and on the flame area, which is increased, on the other hand. In other words, the flame overall burns more slowly but over an extended front. Both opposite effects result in the present case to comparable total heat release rates with and without liquid injection. This conclusion may be however valid only for the present case and could be different in other conditions. The local flame structure itself is clearly modified by the spray, leading to locally stronger and weaker flame parts. This may have a strong impact on ignition and pollutant emissions.

Acknowledgments. This work has been supported by the French $A N R$ agency in the framework of the project ICAMDAC (reference ANR-12-VPTT-07). It was granted access to the High Performance Computing (HPC) resources under the allocations x20152b6139 and x20162b6139 made by GENCI (Grand Equipement National de Calcul Intensif).

\section{References}

1 Robert A., Richard S., Colin O., Martinez L., De Francqueville L. (2015) LES prediction and analysis of knocking combustion in a spark ignition engine, Proc. Combust. Inst. 35, 3, 2941-2948.

2 Pera C., Knop V., Chevillard S., Reveillon J. (2013) Effects of residual burnt gas heterogeneity on early flame propagation and on cyclic variability in spark-ignited engines, Combust. Flame 160, 1020-1032.

3 Lipatnikov A. (2017) Stratified turbulent flames: Recent advances in understanding the influence of mixture inhomogeneities on premixed combustion and modeling challenges, Progr. Energy Combust. Sci. 62, 87-132.

4 He C., Kuenne G., Yildar E., Van Oijen J., di Mare F., Sadiki A. (2017) Evaluation of the flame propagation within an SI engine using flame imaging and LES, Combust. Theory Model. 21, 1080-1113.

5 Fontanesi S., D'Adamo A., Paltrinieri S., Cantore G., Rutland C. (2013) Assessment of the potential of proper orthogonal decomposition for the analysis of combustion CCV and knock tendency in a high performance engine, $S A E$ Technical Paper 2013-24-0031.

6 Fontanesi S., Paltrinieri S., D'Adamo A., Cantore G., Rutland C. (2013) Knock tendency prediction in a high performance engine using LES and tabulated chemistry, $S A E$ Int. J. Fuels Lubr. 6, 1, 98-118.

7 Adomeit P., Lang O., Pischinger S., Aymann R., Graf M., Stapf G. (2007) Analysis of cyclic fluctuations of charge motion and mixture formation in a DISI engine in stratified operation, SAE paper.

8 Forte C., Corti E., Bianchi G.M., Falfari S. (2014) RANS CFD $3 \mathrm{D}$ methodology for the evaluation of the effects of cycle by cycle variation on knock tendency of a high performance spark ignition engine, $S A E$ paper.

9 Moureau V., Lartigue G., Sommerer Y., Angelberger C., Colin O., Poinsot T. (2005) Numerical methods for unsteady compressible multi-component reacting flows on fixed and moving grids, J. Comput. Phys. 202, 710-736.

10 Truffin K., Angelberger C., Richard S. (2015) Using largeeddy simulation and multivariate analysis to understand the sources of combustion cyclic variability in a spark-ignition engine, Combust. Flame 162, 4371-4390.

11 Garcia M., Riber E., Simonin O., Poinsot T. (2005) Comparison between Euler/Euler and Euler/Lagrange LES approaches for confined bluff-body gas-solid flow predictions, Sixth International Conference on Multiphase-Flow.

12 Wehrfritz A., Vuorinen V., Kaario O., Larmi M. (2013) Large Eddy Simulation of high-velocity fuel sprays: studying mesh resolution and breakup model effects for spray A, Atomization Sprays 23, 5, 419-442.

13 Iafrate N., Michel J.B., Cuenot B. (2014) A study of multihole gasoline injector spray dynamics using Large Eddy Simulation, ILASS-Europe.

14 Marmottant P., Villermaux E. (2004) On spray formation, J. Fluid Mech. 498, 73-111.

15 Herrmann M. (2011) On simulating primary atomization using the refined level set grid method, Atomization Sprays 21, 4, 283-301.

16 Lefebvre A.H. (1989) Atomization Sprays, An International Series, Hemisphere Publishing Corporation, Combustion.

17 Ueki H., Ishida D., Naganuma M. (2004) Simultaneous measurements of velocity and size of diesel fuel near nozzle orifice by laser 2 -focus velocimeter with micro-scale probe volume, THIESEL 2004 Conference on Thermo- and Fluid Dynamic Processes in Diesel Engines.

18 Toda H.B., Cabrit O., Balarac S., Bose S., Lee J., Choi H., Nicoud F. (2010) A subgrid-scale model based on singular values for LES in complex geometry, Proceedings of the Summer Program, vol. 192.

19 Misdariis A., Robert A., Vermorel O., Richard S., Poinsot T. (2013) Numerical methods and turbulence modeling for LES of piston engines: impact on flow motion and combustion, Oil Gas Sci. Technol. - Rev. IFP Energies nouvelles 69, 1, 83-105.

20 Vermorel O., Richard S., Colin O., Angelberger C., Benkenida A., Veynante D. (2009) Towards the understanding of cyclic variability in a spark ignited engine using multicycle LES, Combust. Flame 156, 1525-1541.

21 Richard S., Colin O., Vermorel O., Benkenida A., Veynante D. (2006) Towards large eddy simulation of combustion in spark ignition engines, Proc. Combust. Inst., 31, 2, 3059-3066.

22 Colin O., Truffin K. (2011) A spark ignition model for large eddy simulation based on FSD transport equation (ISSIMLES), Proc. (Intl.) Comb. Inst. 33, 2, 3097-3104.

23 Granet V., Vermorel O., Lacour C., Enaux B., Dugue Y., Poinsot T. (2012) Large-Eddy Simulation and experimental study of cycle-to-cycle variations of stable and unstable operating points in a spark ignition engine, Combust. Flame 159, 1562-1575.

24 Robert A. (2014) Simulation aux Grandes Echelles des combustions anormales dans les moteurs downsizes a allumage commande, PhD Thesis, INP Toulouse.

25 Robert A., Richard S., Colin O., Poinsot T. (2015) LES study of deflagration to detonation mechanisms in a downsized spark-ignition engine, Combust. Flame 162, 2788-2807.

26 Metghalchi M., Keck J. (1980) Laminar burning velocity of propane-air mixtures at high temperature and pressure, Combust. Flame 38, 143-154. 
27 Xiouris C., Ye T., Jayachandran J., Egolfopoulos F. (2016) Laminar flame speeds under engine-relevant conditions: uncertainty quantification and minimization in spherically expanding flame experiments, Combust. Flame 163, 270-283.

28 Mannaa O., Mansour M., Roberts W., Chung S. (2015) Laminar burning velocities at elevated pressures for gasoline and gasoline surrogates associated with RON, Combust. Flame 162, 2311-2321.

29 Charlette F., Meneveau C., Veynante D. (2002) A power-law flame wrinkling model for LES of premixed turbulent combustion Part1: non-dynamic formulation and initial tests, Combust. Flame 131, 1-2, 159-180. 